\title{
Quelques données fragmentaires sur l'anthropologie du développement et la coopération en Espagne
}

\section{Danielle Provansal}

\author{
(2) OpenEdition \\ Journals \\ Édition électronique \\ URL : http://journals.openedition.org/apad/389 \\ DOI : 10.4000/apad.389 \\ ISSN : 1950-6929 \\ Éditeur \\ LIT Verlag \\ Édition imprimée \\ Date de publication : 15 mars 1992

\section{Référence électronique} \\ Danielle Provansal, «Quelques données fragmentaires sur l'anthropologie du développement et la \\ coopération en Espagne », Bulletin de l'APAD [En ligne], 3| 1992, mis en ligne le 06 juillet 2006, consulté \\ le 08 septembre 2020. URL : http://journals.openedition.org/apad/389; DOI : https://doi.org/10.4000/ \\ apad.389
}

Ce document a été généré automatiquement le 8 septembre 2020.

Bulletin de l'APAD 


\title{
Quelques données fragmentaires sur l'anthropologie du développement et la coopération en Espagne
}

\author{
Danielle Provansal
}

Quelques antécédents...

1 L'histoire récente de l'Espagne a marqué de son sceau particulier les structures de l'enseignement et de la recherche en ce domaine. En effet, jusqu'en 1970, ce pays était considéré comme pays "en voie de développement, receveur d'aide" selon les critères retenus par les Nations Unies (F.M. Valenzuela ${ }^{1}$ ). Si on ajoute à cela l'isolement relatif de l'Espagne, à la fin du franquisme, et, quelques années plus tard, sa mobilisation autour de la conquête de la démocratie, de la construction d'un cadre étatique respectueux des pluralités historiques et culturelles, l'on comprendra aisément son repliement intellectuel autour de thèmes se rapprochant de sa situation économique et politique. A partir des années soixante dix, les analyses globales de type tiers-mondiste, la mise en relief des rapports de dépendance, à l'échelon mondial ou régional, et les études centrées sur l'ethnicité et sur les phénomènes identitaires ont une place privilégiée dans l'enseignement et la recherche en sciences sociales. En ce qui concerne la coopération, inexistante jusqu'alors, elle débute officiellement en 1977 dans le cadre d'un Fond d'Aide au Développement (FAD). C'est au début des années quatre-vingts qu'est constitué le secrétariat d'État pour la coopération et pour l'Amérique latine (Secretaria de Estado para la Cooperacion y para Iberoamerica-SECIPI-). Cette délimitation régionale s'explique tout d'abord par les liens séculaires qui relient l'Espagne à ses anciennes colonies, mais aussi pour des raisons de politique interne : les fondements de la légitimité du nouvel état des Autonomies - et, en son sein, la délimitation stricte du statut particulier des communautés dites historiques, la Catalogne et le Pays Basque, -s'agencent autour de deux grands axes, l'un qui fait de la monarchie la garante de la démocratie et de l'unité du pays, et l'autre, de type culturel et idéologique, qui cherche à faire de l'hispanité, non seulement un vecteur de rapprochement entre l'Amérique latine et son ancienne métropole mais, aussi et surtout, une "valeur" commune partagée par les différents peuples de la péninsule. 
C'est dans cet esprit qu'un Programme pour le Développement - Cinquième centenaire de la découverte de l'Amérique (Programa ciencia y technologia para el Desarrollo V centenario - CYTED- D-) est mis sur pied en 1984 et établit les bases de la coopération bilatérale entre l'Espagne et l'Amérique latine, fournissant le cadre institutionnel à l'organisme qui va se charger par la suite d'organiser la commémoration du Cinquième Centenaire de la Découverte de l'Amérique (Comision de Comemoracion del quinto Centenario del Descubrimiento de America), à l'aide d'un budget fort important.

La coopération espagnole, aujourd'hui Au niveau de l'État central,

2 La coopération du gouvernement espagnol est essentiellement du ressort du Ministère des affaires étrangères. Le SECIPI en est l'organe dirigeant dont dépendent en premier un certain nombre de directions ou sous-directions, dont la Sous-Direction générale de Relations Economiques Multilatérales et de Développement, (Subdireccion general de Relaciones Economicas Multilaterales y de Desarrollo), la Sous-direction Générale de Coordination de Programmes Communautaires de Coopération (Subdireccion General de Coordinacion de Programas Comunitarios de Cooperacion), en second lieu la Commission Nationale pour la commémoration du Cinquième Centenaire de la Découverte de l'Amérique déjà mentionnée et enfin, l'Agence espagnole de la Coopération Internationale (Agencia espanola de la Cooperacion Internacional - ABCI -) qui regroupe trois instituts de coopération, l'Institut de coopération Iberoaméricaine (ICI), l'Institut de Coopération avec le Monde arabe (ICMA) et l'Institut de Coopération pour le Développement (ICD), qui concerne les pays non inclus dans les deux premiers instituts.

3 Comme on peut le constater, l'entrée de l'Espagne au sein de l'Europe Communautaire signifie un certain élargissement de perspectives au-delà de l'Amérique latine, du moins du point de vue institutionnel. Du point de vue financier, la priorité continue à être réservée aux pays latinoaméricains. En effet, selon les chiffres fournis par le Plan Annuel de Coopération Internationale (PACI) qui est l'instrument financier au service de la coopération espagnole, les prévisions des dépenses de coopération internationale et les prévisions d'aide officielle au développement pour 1991, s'élèvent à 7.520.110.575 pesetas ${ }^{2}$ pour l'Amérique latine et seulement à 3.973.133.88 pesetas pour l'Afrique, soit près de la moitié ${ }^{3}$.

4 Un autre organisme, la Sous-Direction Générale de Coopération de l'Institut de la Jeunesse, dépendant du ministère des Affaires Sociales, encourage la participation des jeunes aux programmes multilatéraux de coopération.

Au niveau des gouvernements autonomes

5 Bien que les gouvernements autonomes n'aient pas de compétences en ce qui concerne les questions internationales, ils peuvent entreprendre certaines campagnes pour encourager l'éducation au développement et la coopération dans certains domaines. A cet égard, le gouvernement catalan a adhéré à l'interdépendance au dialogue Nord-Sud, dans le cadre d'un mouvement désigné sous le nom de "Catalogne solidaire" et regroupant 24 institutions ou organismes. En 1989, le budget de la Généralitat ${ }^{4}$ prévoyait 100 millions de pesetas à l'aide au Tiers Monde.

$\mathrm{Au}$ niveau des Organisations non gouvernementales.

6 Un accord s'est établi entre l'Agence Espagnole de Coopération Internationale et différentes organisations gouvernementales, afin que celles-ci prennent en charge certaines actions dans certaines zones géographiques et pour certains secteurs prioritaires. Leur rôle n'a cessé de s'élargir. Ces organisations, actuellement au nombre 
de 69, se sont fédérées sous le label de Coordinadora de O.N.G. para el desarrollo (Organe de Coordination des O.N.G. pour le Développement). Une partie, variable, du financement des projets de ces organisations provient de fonds publics, et particulièrement du SECIPI. Par exemple, en 1990, le SECIPI a subventionné pour un total de 1.398.698.494 pesetas les projets assumés par des ONG et concernant l'Amérique latine et pour un total de 384.850 .000 ceux qui sont destinés au continent africain. Il est à remarquer que ces organisations sont chaque fois plus nombreuses au sein de l'organisme de coordination. En 1986, elles n'étaient que 22, le montant des subventions que leur accordait le Secrétariat ne s'élevant alors qu'à 32.341 .000 pesetas. Comme on le voit, leur rôle n'a cessé de s'accroître en matière de développement et de coopération. En ce qui concerne les principaux pays africains bénéficiaires, ils sont par ordre d'importance le Cameroun, la Guinée Equatoriale, la République Arabe Sarhaouie Démocratique, le Tchad, le Mozambique, le Burundi, le Ghana et le Cap- Vert ${ }^{5}{ }^{6}$.

De l'aide au développement à la théorie sur le développement

7 Certaines de ces organisations non gouvernementales ont un rôle de formation et d'information et, à travers différentes activités (séminaires, colloques, tables rondes, publications), contribuent à la réflexion sur la problématique du développement. Sans prétention d'exhaustivité, on peut en citer quelques unes particulièrement significatives, quant à leur rôle en ce domaine: à Madrid, l'AIETI (Asociacion de Investigacion y especializacion sobre temas iberoamericanos); le CESAL (Centro de Estudios y Solidaridad con America Latina), le CIDEAL (Centro de Comunicacion, Investigacion y Documentacion entre Europa, Espana y America Latina); le CIPIE (Centro de Investigaciones y Promocion lberoamerica-Europa), IEPALA (Instituto de Estudios Politicos para America Latina y Africa), IPADE (Instituto para la Promocion y Apoyo al Desarrollo), SODEPAZ (Solidaridad para el Desarrollo y la Paz); à Barcelone, le CIDOB (Centro de Informacion y Documentacion Internacional en Barcelona) s'est depuis une quinzaine d'années taillé la part du lion en ce domaine, grâce à son dynamisme et à une excellente organisation. Mon-3, Universitarios para el Tercer Mundo, est une association d'universitaires rattachés à la faculté d'Économie de l'Université de Barcelone qui, périodiquement, organise des cours de formation sur différentes régions du Tiers-monde comme, par exemple, le monde arabe contemporain, le Maghreb, depuis une perspective culturelle et historique, etc. Cette profusion d'organisations et de Centres se consacrant à des tâches d'enseignement sur la problématique du Tiers Monde et des modèles du développement peut paraître étrange mais, en fait, elle répond à un besoin et comble le vide certain qu'en ce domaine l'université espagnole, en tant qu'institution, n'a pas su combler jusqu'à présent. De par sa structure toujours hiérarchique, et surtout en raison de ses modalités de promotion inspirées d'un système hautement compétitif, qui engendre infailliblement des rivalités entre départements et entre les universitaires eux-mêmes, les choix des matières d'enseignement optatives ou des programmes de doctorat se font moins en fonction des besoins et des lacunes existantes que d'intérêts sectoriels, pour ne pas dire sectaires. Le manque de coordination que cette situation engendre- et le manque d'information qui l'accompagne - rendent pratiquement illusoire toute collaboration éventuelle entre les différents spécialistes de la problématique du développement. A la faculté d'économie de l'Université de Barcelone, pionnière en la matière, le sous-développement, le développement et la théorie de la dépendance sont des thèmes abordés depuis plusieurs années. Certains cours d'histoire contemporaine à l'Universidad Computense de Madrid ou à l'Université de Barcelone abordent aussi ces 
questions mais plutôt sous l'angle régional (par exemple en ce qui concerne l'Amérique latine, et dans une moindre mesure, l'Afrique ou le Moyen-Orient). En ce qui concerne le département d'anthropologie sociale et d'histoire de l'Amérique et de l'Afrique de l'Université de Barcelone dont je fais partie, le sous-développement et le développement envisagés sous l'angle d'une pratique articulés sur certains modèles théoriques occupent un certain espace dans les programmes de quelques matières, comme par exemple l'anthropologie économique (Professeur Jesus Contreras), ou encore l'ethnologie régionale (Professeur Dolores Juliano). En ce qui concerne l'anthropologie du développement en Afrique, j'ai abordé cette thématique au sein du cours d'ethnographie africaine ${ }^{7}$ que j'ai donné en 1985 et 1986. Durant l'année académique $87 / 88$ et $88 / 89$, j'ai intitulé le cours sur l'Afrique dont j'avais la responsabilité "Sous-développement, Nationalisme et ethnicité en Afrique", me centrant particulièrement sur le Maghreb et sur l'Afrique de l'Ouest. Depuis l'année académique 89/90, ce cours est plus particulièrement orienté sur les conséquences politiques du sous-développement comme l'indique son intitulé : "Politique et société en Afrique". Dans la même ligne, j'ai organisé, durant cette année académique (91/92), un cours de doctorat sur la construction de l'État africain. Enfin, signalons que l'Université Internationale Menendez Pelayo organise cet été deux cours d'une semaine chacun sur la coopération et le développement, avec la collaboration du Centre d'Etudes Internatioales de l'Université de Barcelone et du Ministère des Affaires Étrangères. C'est malheureusement, et à peu de choses près, la seule information dont je dispose ${ }^{8}$

Cette information peut paraitre fragmentaire et elle l'est en effet, ce qui, à mon avis, n'est pas un hasard. Sous réserve de tout ce qui a pu être ignoré ou oublié, elle reflète d'une certaine manière l'état encore embryonnaire de la recherche et de la dispersion de la réflexion à cet égard. Même si une certaine convergence de points de vue existe de fait entre personnes et disciplines différentes, celle-ci requiert un cadre institutionnel susceptible de rendre fructueuse l'analyse théorique des modèles et des pratiques de développement et d'enrichir ainsi son domaine d'application, autrement dit la pratique de terrain elle-même, par le biais de la coopération ${ }^{9}$.

\section{NOTES}

1.Anuario CIDOB, Barcelona, 1990

2.100 pesetas équivalent approximativement à cinq francs.

3.L'aide officielle au développement connaît un accroissement de 139 \% en 1989, passant de 124,9 millions de dollars en 1988 à 194,3 millions de dollars en 1989.

L'assistance technique connaît un essor encore plus spectaculaire chiffré à $458 \%$ de son montant : elle passe de 24,6 millions de dollars à 137,2 millions de dollars entre 1988 et 1989 (ibid).

4.Gouvernement autonome catalan.

5.Ne sont mentionnés que les pays au bénéfice d'un projet de développement supérieur à 5 millions de pesetas. 
6.Anuario CIDOB, 1991.

7.Il s'agit d'une matière optative de deuxième cycle.

8.Sachant par ouie dire qu'il existait un cours sur l'anthropologie du Développement donné par le Département de sociologie et d'anthropologie sociale de l'Université Autonome de Madrid et ayant pris contact avec son responsable, je n'ai pu, malgré mes demandes réitérées, obtenir le moindre renseignement sur le titre de ce ou ces (?) cours et sur son programme. Il en a été de même pour d'autres centres universitaires, plus précisément à Tenerife et à Séville qui parait-il, aborderaient ces thématiques. 9.Je tiens à remercier Joseph Ribera, Directeur du CIDOB et Remei Sipi également du CIDOB pour leur précieuse collaboration.

\section{AUTEUR}

\section{DANIELLE PROVANSAL}

Professeur titulaire, Université de Barcelone 